\title{
LETTERS
}

\section{Subsequent COPD and lung cancer in patients with autoimmune disease}

\section{To the Editors:}

Autoimmune diseases can be defined as clinical syndromes caused by the activation of T-cells or B-cells, or both, in the absence of an ongoing infection or other discernible cause [1]. These cells recognise specific foreign antigens but even in healthy individuals they possess a low-level of autoreactivity towards own antigens. Autoreactivity is increased in autoimmune diseases either systemically (as in rheumatoid arthritis) or organ-specifically (Graves' disease and type 1 diabetes mellitus). These diseases are diagnosed by clinical symptoms and by specific types of autoantibodies. The prevalence of autoimmune diseases ranges from $5-10 \%$ of the population in Western countries, including common diseases, such as rheumatoid arthritis and autoimmune thyroiditis, and many rare ones [2]. Epidemiological studies have demonstrated that genetic factors are crucial determinants of susceptibility to autoimmune disease [1]. For many of these diseases a familial clustering is reported. However, even in a genetically predisposed person, some trigger, an environmental exposure or a change in the internal environment, is usually required to precipitate autoreactivity [3]. Smoking is an environmental risk factor for some autoimmune diseases, including rheumatoid arthritis, systemic lupus erythematosus, Graves' disease and primary biliary cirrhosis, but for many other autoimmune diseases data on smoking are either lacking or controversial.

Chronic obstructive pulmonary disease (COPD) is a slowly progressive disease characterised by irreversible airflow limitation, which is caused by chronic inflammatory immune response to inhaled gases and particles [4]. It may affect up to $10 \%$ of the population. In the early phase, the inflammatory process is located in central airways but in progressive disease the distal airspaces are enlarged because of destruction of airway walls. In some cases only small airways are obstructed with minor impairment in lung function tests. Increasing lines of evidence show that autoimmune mechanisms are involved in COPD [4]. $\alpha$-Antitrypsin deficiency is an established genetic risk factor of emphysema, but its prevalence is $<0.1 \%$ in the population and thus it will account for a small proportion of COPD [5]. Tobacco smoking is the single most common risk factor for COPD but even many other environmental factors are known [5]. In smokers of $>40$ pack-yrs, the prevalence of COPD has been reported to be $>50 \%$ [6]. Smoking is a shared risk factor for COPD and lung cancer.

In the present paper, we examine associations of autoimmune diseases, COPD and lung cancer, first to test for a possible shared aetiology between autoimmune disease and COPD, and secondly to test for a possibility that smoking may be a risk factor of autoimmune diseases.
The autoimmune disease and COPD research database, used for this study, was constructed by linking several national Swedish registers, as has been described earlier [7, 8]. Data on autoimmune diseases and COPD were obtained from the Swedish Hospital Discharge Register that records complete data on all discharges with dates of hospitalisation and diagnoses in some regions since 1964 and nationwide since 1986; cancer data were retrieved from the Swedish Cancer Registry. All linkages were performed by the use of an individual national identification number that is assigned to each person in Sweden for their lifetime. This number was replaced by a serial number for each person in order to provide anonymity.

The International Classification of Diseases codes used have been previously described [7, 9]. Risk of COPD and lung cancer was determined for persons who had been hospitalised for autoimmune disease any time between 1964 and 1999 compared with persons who had not been hospitalised for autoimmune disease. Person-yrs were calculated for COPD and lung cancer from start of follow-up on January 1, 2000 until hospitalisation for COPD or lung cancer, death, emigration or closing date on December 31, 2006. Patients hospitalised for COPD or diagnosed with lung cancer prior to the year 2000 were excluded. The reasons for starting the follow-up in the year 2000 were to allow for uniform diagnostics and to exclude the possible lead time bias that hospitalisation and the resulting medical examinations may cause $[8,9]$. This decision implied that for some patients the time difference between hospitalisations for autoimmune disease and COPD/lung cancer was long, which could lead to increased risks. Standardised incidence ratios (SIRs) were calculated as the ratio of observed to expected number of cases. The expected number of cases was calculated for age (5-yr groups), sex, period (5-yr groups), region and socioeconomic status-specific standard incidence rates.

In table 1, 29 autoimmune diseases are shown with at least five patients diagnosed in each with COPD; four other diseases are not shown because only one or no COPD was diagnosed. The risk of COPD was increased in patients hospitalised for 18 different autoimmune diseases (the total patient number was 297,300), among which the highest SIRs were observed for polyarteritis nodosa (5.69), primary biliary cirrhosis (4.79), pernicious anaemia (4.04), polymyalgia rheumatic (3.77), Addison disease (3.56), myasthenia gravis (3.27), chronic rheumatic heart disease (3.13) and systemic sclerosis (3.08) (all over 3.00). The SIR for all included autoimmune diseases was 1.99 in 1,474 COPD patients. The SIR for type 1 diabetes was decreased to 0.42 .

According to table 1, high risks of lung cancer were observed in systemic sclerosis (4.45), discoid lupus erythematosus (4.24) 


\begin{tabular}{|c|c|c|c|c|c|c|c|c|}
\hline \multirow[t]{2}{*}{ Autoimmune disease } & \multicolumn{4}{|c|}{ COPD } & \multicolumn{4}{|c|}{ Lung cancer } \\
\hline & Person yrs & Obs. & SIR & $95 \% \mathrm{Cl}$ & Person yrs & Obs. & SIR & $95 \% \mathrm{Cl}$ \\
\hline Addison's disease & 5195 & 10 & 3.56 & $1.69-6.57$ & 6353 & 3 & 0.87 & $0.16-2.57$ \\
\hline Ankylosing spondylitis & 21279 & 41 & 1.72 & $1.23-2.33$ & 24116 & 11 & 0.72 & $0.36-1.29$ \\
\hline Behçet's disease & 10627 & 9 & 0.94 & $0.43-1.80$ & 10867 & 7 & 1.15 & $0.45-2.37$ \\
\hline Celiac disease & 25277 & 12 & 0.59 & $0.31-1.04$ & 31288 & 3 & 1.26 & $0.24-3.74$ \\
\hline Chronic rheumatic heart disease & 20121 & 85 & 3.13 & $2.50-3.88$ & 20976 & 20 & 0.93 & $0.57-1.44$ \\
\hline Crohn's disease & 117202 & 132 & 1.18 & $0.99-1.40$ & 130065 & 60 & 1.05 & $0.80-1.35$ \\
\hline Diabetes mellitus type 1 & 80525 & 24 & 0.42 & $0.27-0.62$ & 89180 & 1 & 0.48 & $0.00-2.77$ \\
\hline Localised scleroderma & 14138 & 21 & 1.36 & $0.84-2.08$ & 14398 & 9 & 1.20 & $0.55-2.30$ \\
\hline Multiple sclerosis & 33398 & 45 & 1.03 & $0.75-1.39$ & 35891 & 18 & 0.86 & $0.51-1.36$ \\
\hline Myasthenia gravis & 50058 & 111 & 3.27 & $2.69-3.94$ & 49010 & 46 & 1.02 & $0.74-1.36$ \\
\hline Pernicious anaemia & 9954 & 25 & 4.04 & $2.61-5.97$ & 9980 & 10 & 0.94 & $0.45-1.73$ \\
\hline Polyarteritis nodosa & 11688 & 45 & 5.69 & $4.15-7.62$ & 11824 & 9 & 0.76 & $0.35-1.46$ \\
\hline Polymyalgia rheumatica & 41301 & 146 & 3.77 & $3.18-4.44$ & 42628 & 36 & 0.90 & $0.63-1.25$ \\
\hline Polymyositis/dermatomyositis & 2821 & 6 & 1.93 & $0.69-4.23$ & 3484 & 8 & 3.92 & $1.67-7.76$ \\
\hline Primary biliary cirrhosis & 2467 & 8 & 4.79 & $2.05-9.49$ & 2861 & 2 & 0.89 & $0.08-3.27$ \\
\hline Psoriasis & 46377 & 101 & 2.44 & $1.99-2.97$ & 50146 & 44 & 1.33 & $0.97-1.79$ \\
\hline Rheumatic fever & 13327 & 28 & 2.05 & $1.36-2.97$ & 13786 & 10 & 1.09 & $0.52-2.02$ \\
\hline Rheumatoid arthritis & 64834 & 181 & 2.57 & $2.21-2.97$ & 103101 & 118 & 1.48 & $1.23-1.77$ \\
\hline
\end{tabular}

Obs.: observed; SIR: standardised incidence ratios. Numbers shown in bold font: $95 \%$ Cls do not overlap with 1.00 .

and polymyositis/dermatomyositis patients (3.92). Also systemic lupus (2.72) and rheumatoid arthritis (1.48) were associated with lung cancer. Overall, 617 patients with an SIR of 1.18 were diagnosed with lung cancer.

The use of the Hospital Discharge Register to identify patients with autoimmune diseases offers access to a nationwide patient pool with a reasonably high diagnostic accuracy; the discharge diagnoses are often delivered by specialists during extended examinations in the clinic. However, the degree of hospitalisation for the covered autoimmune diseases probably vary extensively depending on the seriousness of the condition and alternative forms of treatment, as we have discussed for some of the covered diseases elsewhere [8, 9]. For some diseases most patients have been hospitalised in the course of the disease, including hyperthyroidism, Crohn's disease, ulcerative colitis, type 1 diabetes and rheumatoid arthritis. For these, the estimated risks for COPD appear generally valid. For other autoimmune diseases with a lower degree of hospitalisation, such as hypothyroidism, the data may be valid only for the hospitalised portion of the patient population.

In the present study, 18 autoimmune diseases were associated with COPD and five with lung cancer. The diseases associated with COPD included rheumatoid arthritis, systemic lupus erythematosus, Graves' disease, primary biliary cirrhosis, polymyalgia rheumatica and psoriasis, for which smoking is a known risk factor $[3,10]$. However, the remarkable lack of excess lung cancer in patients with the five highest risk autoimmune diseases (polyarteritis nodosa, pernicious anaemia, Addison's disease, myasthenia gravis and chronic rheumatic heart disease) is a strong indication of an autoimmune contribution to COPD. It was also interesting that the associations of polymyositis/dermatomyositis with lung cancer showed no significant (although increased) risks for COPD. The risks for lung cancer in patients with systemic sclerosis, discoid lupus erythematosus and polymyositis/ dermatomyositis were remarkably high, around 4 or more, 
which may suggest shared predisposition to these autoimmune diseases and lung cancer. Advice on smoking control would be appropriate for patients with these diseases.

In conclusion, the increased risk of COPD but not lung cancer in patients with many autoimmune diseases suggests that the defined autoimmunity may predispose to COPD. Another possibility is that smoking is a risk factor for some of these autoimmune diseases and the effects on lung cancer cannot be recognised because of the small number of cases. This possibility could not be ruled out because data on smoking were lacking. The four-fold risks of lung cancer in patients with systemic sclerosis, discoid lupus erythematosus and polymyositis/dermatomyositis may indicate susceptibility to lung cancer, which should be considered in the counselling of these patients.

\section{K. Hemminki*,\# ${ }^{*}$ X. Liü ${ }^{\#}$ J. Ji ${ }^{\#}$, K. Sundquist ${ }^{\#}$ and J. Sundquist ${ }^{\#, \text { 厂 }}$}

*Division of Molecular Genetic Epidemiology, German Cancer Research Center (DKFZ), Heidelberg, Germany, "Center for Health Care Research, Lund University, Malmö, Sweden, and 'Stanford Prevention Research Center, Stanford University School of Medicine, Stanford, CA, USA.

Correspondence: K. Hemminki, DKFZ, Im Neuenheimer Feld 580, D-69120 Heidelberg, Germany. E-mail: k.hemminki @dkfz.de

Support Statement: Supported by the Swedish Council for Working Life and Social Research (Stockholm, Sweden), the Swedish Cancer Society (Stockholm, Sweden) and Deutsche Krebshilfe (Bonn, Germany).
Acknowledgements: The used database was created by linking registers maintained at Statistics Sweden (Stockholm, Sweden) and the Swedish Cancer Registry.

\section{REFERENCES}

1 Zhernakova A, van Diemen CC, Wijmenga C. Detecting shared pathogenesis from the shared genetics of immune-related diseases. Nat Rev Genet 2009; 10: 43-55.

2 Forabosco P, Bouzigon E, Ng MY, et al. Meta-analysis of genomewide linkage studies across autoimmune diseases. Eur J Hum Genet 2009; 17: 236-243.

3 Costenbader $\mathrm{KH}$, Karlson EW. Cigarette smoking and autoimmune disease: what can we learn from epidemiology? Lupus 2006; 15: 737-745.

4 Cosio MG, Saetta M, Agusti A. Immunologic aspects of chronic obstructive pulmonary disease. N Engl J Med 2009; 360: 2445-2454.

5 Walter R, Gottlieb DJ, O'Connor GT. Environmental and genetic risk factors and gene-environment interactions in the pathogenesis of chronic obstructive lung disease. Environ Health Perspect 2000; 108: Suppl. 4, 733-742.

6 Jyrki-Tapani K, Sovijarvi A, Lundback B. Chronic obstructive pulmonary disease in Finland: prevalence and risk factors. COPD 2005; 2: 331-339.

7 Hemminki K, Li X, Sundquist K, et al. Familial risks for chronic obstructive pulmonary disease among siblings based on hospitalizations in Sweden. J Epidemiol Community Health 2008; 62: 398-401.

8 Hemminki K, Li X, Sundquist J, et al. Familial associations of rheumatoid arthritis with autoimmune disorders and related conditions. Arthritis Rheum 2009; 60: 661-668.

9 Hemminki K, Li X, Sundquist K, et al. Familial risks for asthma among twins and other siblings based on hospitalizations in Sweden. Clin Exp Allergy 2007; 37: 1320-1325.

10 Cimmino MA, Zaccaria A. Epidemiology of polymyalgia rheumatica. Clin Exp Rheumatol 2000; 18: S9-S11.

\section{Clinical features of secondary pulmonary alveolar proteinosis: pre-mortem cases in Japan}

\section{To the Editors:}

Pulmonary alveolar proteinosis (PAP) is a rare syndrome that predominantly affects the lungs, and is characterised by the accumulation of surfactant lipids and proteins in the alveoli and terminal airways [1]. In 1999, we published findings that high levels of granulocyte-macrophage colony-stimulating factor (GM-CSF) neutralising autoantibodies were detected specifically in patients with idiopathic PAP [2]. Recently, as a result of the excellent sensitivity and specificity of GM-CSF autoantibody assays in identifying this form of PAP [3], it has been proposed that the nomenclature for this condition should be changed to "autoimmune PAP" rather than "idiopathic $\mathrm{PAP}^{\prime \prime}$ [4]. Secondary or hereditary PAP is not associated with
GM-CSF autoantibodies but develops as a consequence of a separate underlying disorder or genetic background [5]. Recently, we demonstrated that the characteristic high-resolution computed tomography (HRCT) findings in secondary PAP are distinct from those in autoimmune PAP [6]. However, physicians may not suspect secondary PAP, even when encountering unexplained pulmonary opacities on chest radiograph or computed tomography in patients with pre-existing haematological or infectious diseases, since there is little information available on the clinical features. Here, we outline the clinical features of adult-onset secondary PAP based on our database of 40 pre-mortem cases in which serum GM-CSF autoantibodies were negative, and compare them with other cases in the literature. 